Aus dem Sanatorium Hoppegarten. (Leiter: DDr. Leubuscher und Wagner.)

\title{
Therapeutische Versuche mit Phosphor bei Epileptikern.
}

\section{Von Dr. Paul Leubuscher.}

Bis in die neueste Zeit hinein dominierte bei der Behandlung der Epilepsie besonders in Deutschland und Frankreich die innere Therapie. Als Hauptheilmittel wurde und wird das Brom betrachtet. In den letzten Jahren hat man über die Bromwirkung auch theoretisch etwas eingehendere Erwägungen angestellt und gelangte, wie selbstverständlich, dazu, den gesamten Salzstoffwechsel als maßgebend für die Anschauung der physiologischen Bromwirkung anzusehen.

Ausgehend von den Gesetzen der chemischen Umsetzung in Salzlösungen, fand man die Bromwirkung im umgekehrten Verhältnis stehend zur Chloraufnahme. Hieraus ergeben sich die bekannten Vorschläge einer speziellen Behandlungsmethode, beginnend von Richet Toulouse bis neuerdings $W$ yes und Ulrich. Letzterer erblickt bereits lediglich in der starken Verminderung der Chloraufnahme ein Heilmittel für die epileptischen Erscheinungen.

Wenn man nun auch zugeben muß, daß die Veränderung der Salzzuführung, sei es durch Weglassen des Chlors, sei es durch Aufnahme von erheblichen Mengen Bromsalz in vielen, besonders frischen Epilepsiefällen eine bedeutende Wirkung ausübt, so sind wir doch noch weit entfernt von der Möglichkeit, überhaupt durch Verdrängung des Chlors eine Heilung der meisten Epilepsieerkrankungen herbeizuführen.

Wohl wird berichtet, daß durch geschickte und systematische Brombehandlung die Anfälle in ihrer Zahl und Stärke herabgesetzt werden, und vielleicht kann man annehmen, wie viele Autoren es tun, daß nach langer künstlich herbeigeführter Unterdrückung der Krampfanfälle überhaupt die Disposition der Hirnrinde für krampfartige Explosionen verschwindet. Doch ist die Zahl der Fälle, in denen letzteres wirklich für lange Zeit oder für immer eingetreten ist, nachdem die medikamentöse Behandlung selbst ausgesetzt ist, überaus gering.

Die Nebenwirkungen des Broms sind, wie man betonen muß, abgesehen von der Akne, ganz außerordentlich erheblich. Es ist nicht gleichgültig, ob im Stoffwechsel das Chlor durch Brom ersetzt wird, oder ob dauernd eine ungenügende Menge Chlor kreist und wirkt. Der Brommensch, der auf diese Weise entsteht, ist immerhin ein künstliches Produkt und auf alle Fälle pathologisch, wenn er auch keine oder nur wenige Anfälle hat.

Wohl muß man zugeben, daß wir in vielen Fällen des Broms nicht entraten können, daß es eine Reihe von Epilepsiefällen gibt, bei denen die gefahrdrohende Häufung der Anfälle und die Möglichkeit, sie durch Brom zu vermindern, alle anderen Bedenken schwinden läßt und uns unbedingt zu dieser Behandlung zwingt. Wir müssen uns jedoch dabei immer bewußt sein, daß wir nur künstlich die Reizbarkeit der Hirnrinde herabsetzen und eigentlich nicht die Krankheit selbst wesentlich beeinflussen.

Es bleibt nun noch ein nicht geringer Teil von Epileptikern übrig, bei denen Brombehandlung oder Chlorentziehung effektiv gar keine heilende Wirkung ausübt, wie dies in jeder größeren Epileptikeranstalt seit langem beobachtet worden ist. Wir müssen aber bei der Behandlung von der Vorstellung ausgehen, die auch Binswanger mehrfach ausgesprochen hat, daß die Epilepsie wohl in der Art ihrer Krankheits. äußerungen als einheitlich angesehen werden kann, daß aber diesem klinisch einheitlichen Bild die mannigfachsten Ursachen zugrunde liegen können.

So wird es uns klar werden, daß das Brom eben nicht als das gegebene Behandlungsmittel für jeden Epilepsiefall anzusehen ist, daß es nur in wenigen Fällen die noch geheimnisvollen Ursachen der Krankheit fassen kann.

Wenn wir annehmen, daß Gefäßveränderungen oder störungen wesentlich bei der Pathogenese der Epilepsie sind, so ist es berechtigt zu versuchen, Mittel anzuwenden, die auf das Gefäßsystem wirken.

So haben sich Valeriana-Präparate, Digitalis und Adonis in dem bekannten Bechterewschen Mittel ihren Platz bereits erobert. Auch 
mit neueren Präparaten, z. B. Vasotonin, sind Versuche, unter anderm auch von uns, auf Anregung von $\mathrm{Ziehen}$ gemacht worden.

A u toin toxikationen, vom Darm ausgehend, spielen ohne Zweifel die größte Rolle bei der Auslösung von Anfällen. Reinigung des Darmes ist daher das erste Mittel bei Serien und Status. In dieser Weise mögen der besonders in England immer noch beliebte Borax und ähnliche Arzneien wirken.

Sowohl die Berücksichtigung des Gefäßsystems, wie auch das Streben, Autointoxikationen zu verhindern oder $\mathrm{zu}$ beseitigen, kommen nun dem Gedanken näher, die auslösenden Momente, die für bestimmte Fälle von Epilepsie maßgebend sein mögen, zu bekämpfen. Immerhin sind wir, wenn wir die Veränderung des Salzstoffwechsels mit ihren guten und schlechten Seiten als symptomatisch betrachten und die Erfolge der letztgenannten Behandlungsmethode noch immer nicht als erheblich ansehen können, der sozial so eingreifenden Krankheit gegenüber in einer schlechten Lage. Es erscheint daher ebenso berechtigt wie geboten, jedes Mittel zu versuchen, das eine gewisse physiologische Ueberlegung für sich hat, ganz besonders, wenn die empirisch bereits damit erzielten Erfolge eine Wirksamkeit zu gewährleisten scheinen.

So hat man Versuche mit Organpräparaten, dem Pohlschen Cerebrin, gemacht, für das Lion ganz besonders eingetreten ist, so berichten weiter amerikanische Aerzte, Self und Spangler, und hiel in Deutschland Fackenheim von wesentlichen Erfolgen, die sie mit dem Crotalin, dem Gift der Klapperschlange, erzielt haben.

Ich habe nun vor ungefähr zwei Jahren begonnen, einen Teil der Patienten unseres Sanatoriums mit Phosphor zu behandeln. Anregend wirkte auf die Auswahl dieses Mittels die Einwirkung des Phosphors auf die Tetanie. Wenn auch die klinische Stellung der Tetanie entfernt ist von der Epilepsie, so ist der Gedanke, daß vielleicht ähnliche physiologische Momente bei dieser Krankheit mitwirken, doch nicht ganz von der Hand zu weisen. Auch lag ein Fall von Gerhartz vor, der mit großem Erfolg ein an Epilepsie leidendes Kind mit Phosphor behandelt hat. Auf letzteren Fall machte mich unser damaliger Assistenzarzt, Herr Dr. Goldsch mid t, aufmerksam.

Im März/April 1911 begann ich einer Reihe von Patienten Phosphor in öliger Lösung 0,1 : 1000,0 oleum olivarum zu geben. Im nächsten Jahre habe ich dies weiter fortgesetzt, sodaß ich jetzt über 9 Patienten verfüge, über deren Behandlung und Ergehen ich berichten kann. Diese Patienten sind in der Behandlungsperiode nur mit Phosphoröl behandelt worden, ohne daß ein anderes Mittel angewandt wurde. Bei 6 Patienten beträgt die Behandlungszeit etwa 15 Monate.

Da alle Patienten auch vor der Behandlung lange Zeit in unsere Anstalt waren und genau beobachtet wurden, bin ich in der Lage, einen sicheren Vergleich der Anzahl der Krampfanfälle in der Vorperiode und der Anzahl der Krampfanfälle in einer gleich langen Behandlungsperiode zu ziehen

Kurz möchte ich jetzt die einzelnen Fälle erwähnen.

Fall 1. Me., 28 Jahre alt. Der Kranke soll seit dem 12. Lebensjahr an epileptischen Krampfanfällen leiden. In der Jugend soll eine Gehirnentzündung aufgetreten sein. Es besteht erheblicher Schwach sinn. Am 20. Juni 1910 wurde der Kranke in die Anstalt aufgenommen. Von diesem Zeitpunkt bis zum 19. April 1911 betrug der monatliche Durchschnitt der Anfälle 12,9. Am 19. April 1911 begann die Phosphorbehandlung. Bis zum 1. August 1912 stieg der monatliche Durchschnit der Anfälle auf 14,3.

Fall 2. He., 30 Jahre alt. Zahlreiche Krampfanfälle seit dem 12. Lebensjahr. Schwachsinn. Körperlich nichts wesentlich Krankhaftes. Monatlicher Durchschnitt der Zahl der Anfälle in der Vorperiode von 15 Monaten 15,5.. Monatlicher Durchschnitt während der Behandlun mit Phosphor von 15 Monaten 14,6. Dabei ist zu berücksichtigen, daß seit Februar 1912 die Krämpfanfälle bedeutend nachließen und mit 6 im April überhaupt ihren niedrigsten Stand seit 1909, wo der Kranke in unsere Behandlung $\mathrm{kam}$, erlangten

Fall 3. Bi., 20 Jahre alt; körperlich sehr kräftig entwickelt. Schwachsinn mäßigen Grades. Die Krämpfe bestehen seit dem 4. Lebensjahre, nach Angabe der Mutter soll damals eine Kopfverletzung vorangegangen sein. Es ist über dem linken Auge noch eine $6 \mathrm{~cm}$ lange Narbe vorhanden Die Krampfanfälle sind bisher immer sehr schwer gewesen, die Bevorzugung einer Seite ist nicht nachweisbar. In den Jahren 1906 bis Oktober 1908 kann eine Brombehandlung von 3 bis 4,0 Bromkali mit Sicherheit nachgewiesen werden.

Während dieser Brombehandlung war der monatliche Durchschnitt der Krampfanfälle ungefähr 13 bis 14 . Nachdem das Brom aus gesetzt war, blieb seitdem die Anzahl der Anfälle fast unverändert. Sie betrug vom 1 . Januar 1910 bis zum 28. März 1911 im Durchschnit 14,2. Vom 28. März 1911 setzte die Phosphorbehandlung ein, und bis zum 1. August 1912 betrug nun der monatliche Durchschnitt 11,2 .

Fall 4. H., 30 Jahre alt, erblich belastet. Körperlich nichts Krankhaftes. Die Anfälle sind schwer, zeitweise treten auch Erregungs- und Verwirrungszustände auf. Monatlicher Durchschnitt der Zahl der Anfälle in der Vorperiode von 15 Monaten 12,6, außerdem 1,2 leichte Schwindelanfälle. Monatlicher Durchschnitt während der Behandlungsperiode mit Phosphor 9,7. Allerdings stieg Ende 1911 die Anzahl der leichten Schwindelanfälle. Sie ging jedoch in diesem Jahr auch wesentlich zurück.

Fall 5. F., 19 Jahre alt, erblich belastet. Es besteht mäßiger Schwachsinn. Körperlich außer einer alten, tiefen Narbe an der Stirn nichts Krankhaftes. Im Jahre 1903 Schädelbruch durch Fall vom Wagen. Seit 1904 sind zahlreiche Krampfanfälle aufgetreten, die völlig typisch, nicht im Sinne einer Jackson-Epilepsie verlaufen. In den letzten Jahren keine Therapie. In der Zeit vom 1. Januar 1910 bis 19. April 1911 während 16 Monaten im monatlichen Durchschnitt 119,2 Anfälle. Seitdem wurde Phosphor gegeben, und die Anzahl der Anfälle bis zum 1. August 1912 stellte sich auf 73,2 Anfälle im monatlichen Durchschnitt. Außerdem wurde bemerkt, daß die Anfälle nicht unwesentlich leichter geworden sind.

Fall 6. D., 20 Jahre alt. Krampfanfälle bestehen seit dem 5. Lebensjahre. Der Kranke ist ziemlich schwachsinnig, zeitweise treten Verwirrungszustände auf. Körperlich außer einer alten Augenerkrankung nichts wesentlich Krankhaftes. Patient hatte in der Vorperiode vom 1. Januar 1910 bis 19. April 1911, also während 16 Monaten, 12,6 Anfälle im monatlichen Durchschnitt, vom 19. April 1911, als die Phosphorbehandlung begann, bis zum 1. August 1912 betrug der monatliche Durchschnitt 7 Anfälle.

Fall 7. M., 24 Jahre alt, erblich schwer belastet. Krampfanfälle und Erregungszustände. 1899 zunehmende Demenz. Dauernd unzufrieden und mürrisch. Die Anzahl der Krampfanfälle betrug vom 1. Dezember 1911 bis 5 . April 1912 im monatlichen Durchschnitt 21. Am 5. April 1912 begann die Phosphorbehandlung, von dann an bis zum 1. August 1912 betrug der monatliche Durchschnitt der Anfälle 16.

Fall 8. B., 24 Jahre alt, erblich belastet. Vor 8 Jahren die ersten Schwindelanfälle, seit 6 Jahren Krampfanfälle. Demenz. Stark gesteigerte Sehnenreflexe. Innere Organe gesund. Es besteht Neigung zu Magenverstimmung. Patient erhielt vom 10. April 1912 ab Phosphor. Die Anzahl der Krampfanfälle in den 7 Monaten vor der Therapie betrug in monatlichem Durchschnitt 7 und 1,3 leichte Schwindelanfälle. Seit der Therapie betrug die Anzahl der Krampfanfälle im monatlichen Durchschnitt 2,3 und 2,7 leichte Schwindelanfälle.

Fall 9. Pa., 17 Jahre alt, erblich belastet. Hereditäre Lues. (Wa.R. positiv.) Körperlich zurückgeblieben. Herzfehler. Rechts alte Hornhaut. erkrankung. Erheblicher Schwachsinn. Krämpfe seit dem 7. Lebensjahre. Vor der Behandlung vom 1. Januar 1910 bis 23. März 1911 im monatlichen Durchschnitt 53,2 Anfälle. Am 23. März 1911 begann die Phosphorbehandlung, und es stellte sich der monatliche Durchschnitt während 16 Monaten bis 1. August 1912 auf 16 Anfälle.

Ich habe von den mit Phosphor behandelten Patienten diejenigen Fälle beschrieben, bei denen sowohl in der Vorperiode wie während der Behandlung außergewöhnliche Ereignisse nicht eingetreten sind. Die $\mathrm{Pa}$ tienten, bei denen länger dauernde Erregungszustände, körperliche Erkrankungen wesentlicher Art. Verweigerung des Mittels etc. in Frage kommen, sind natürlich nicht init aufgeführt. Die beschriebenen Fälle sind dauernd in unserer Anstalt gewesen, die Krampfanfälle sind in beiden Perioden in genau gleicher Art notiert worden.

Bei der Auswahl der mit Phosphor behandelten Patienten habe ich fast nur genuine Epilepsiefälle berücksichtigt. Auch bei den Kranken, bei denen, wie angeführt, ein Trauma mitgewirkt haben soll, liegt der ganzen Art der Krankheit und der Anamnese nach die Vermutung nahe, daß es sich um genuine Epilepsie handelt, bei der die Krämpfe durch das Trauma wohl nur verstärkt oder allenfalls frühzeitig ausgelöst sind.

Spätepilepsie, insbesondere Alkoholepilepsie, habe ich bisher noch nicht mit Phosphor behandelt.

Wenn wir nun die oben angeführten Fälle năher betrachten, so sehen wir, daß bei dem Kranken Me. die Krämpfe sich an Zahl etwas vermehrt haben.

In drei Fällen ist der Rückgang nicht allzu bedeutend, d. h. von 15,5 auf 14,6 , von 14,2 auf 11,2 und von 12,6 auf 9,7 . Immerhin ist auch hier, da es sich um einen längeren Zeitraum der Beobachtung handelt, 15 bis 16 Monate, die Verminderung der Anfälle um 10 bis $20 \%$ deutlich

In drei weiteren Fällen, bei den Kranken F., D. und Mi., fiel die Anzahl der Krampfanfälle von 119,2 auf 73,2 , von 12,6 auf 7 und von 21 auf 16. Das bedentet eine Verninderung um ungefähr 30 bis $40 \%$ es ist also ein wesentlicher Fortschritt erzielt worden.

Bei dem Kranken B. ging die Anzahl der Krampfanfälle von 7 auf 2,3 zurück, also um etwa $65 \%$, wenngleich die kleinen Schwindelanfälle von 1,3 bis auf 2,7 stiegen.

Bei dem Kranken P., der übrigens der jüngste von allen ist, gingen die Krampfanfälle von 53,2 auf 16 zurück, sie erreichten noch nicht einmal den dritten Teil der vorher beobachteten Anfälle.

Sämtliche Kranke haben die Behandlung im übrigen körperlich sehr gut vertracon. Das Körpergewicht hat sich in der ganzen Zeit un gefähr auf der gleichen Höhe gehalten. Bei einigen schien es, als ob auch psychisch eine gewisse Besserung eingetreten war, jedoch sind hier die 
Unterschiede nicht erheblich genug, $11 \mathrm{~m}$ besonders hervorgehoben zu werden.

Ich erwähne noch, daß die erzielten Besserungen bei fast allen Kranken auch nach Aussetzen der Phosphorbehandlung und ohne daß neuere Mittel, speziell Brom von neuem gegeben wurde, angehalten haben. Da imnerhin die Zeit seit denı Aussetzen des Mittels bis zur Niederschrift dieser Arbeit nur kurz ist, kann ich daraus noch keine sicheren Sohlüsse ziehell.

Auffällig war, daß bei dem Kranken F. gleich nach Aufhöreı des Phosphors die Zahl der Anfälle wieder sehr stieg, um dann zur Norm der letzten Monate zurückzukehren.

Wenn nun anch mit dieser Behandlung keine tatsächlichen Heilungen erzielt sind, so muß man berücksichtigen, daß es sich um ganz besonders schwere Epilepsieerkrankungen mit sehr zahlreichen, bereits seit vielen Jahren bestehenden Anfällen handelte. Immerhin ist es sicher, daß hier ein keineswegs unbedeutender therapeutischer Einfluß des Phosphors auf Krampfanfälle vorliegt. Man kann vielleicht annehmen, daß es sich um Oxydationsvorgänge handelt, die krampfauslösende Stoffwechselprodukte vernichten. Ein Oxydationsprozeß würde einer von Pflüger einmal ausgesprochenen Auffassung der Phosphorwirkung im mensohlichen Organismus entsprechen.

Fin näheres Eingehen auf die physiologische Möglichkeit der Phosphorwirkung und die Vorgänge, die sich dabei abspielen können, möchte ich mir, besonders nach einer weiteren Vermehrung des klinischell Materials, noch vorbehalten.

$\mathrm{Ob}$ diese gefundene Wirkung auch bei epileptisch Erkrankten leichterer Art und solchen, die andern Altersstufen angehören, eintritt, ist noch festzustellen. 